\title{
Die Massenkonzentration der alkalischen Knochen- phosphatase ist der Bestimmung von Osteocalcin bei der Diagnose von Knochenmetastasen überlegen
}

\author{
Determination of Serum Bone Alkaline Phosphatase Mass Concentrations is Superior to \\ Osteocalcin in the Detection of Metastatic Spread into Bone
}

\author{
W. Withold ${ }^{1,2}$
}

Zusammenfassung: Bei 73 Tumorpatienten wurde die diagnostische Validität von zwei klinisch-chemischen Meßgrößen des Knochenanbaus (Osteocalcin, alkalische Knochenphosphatase) miteinander verglichen. Die Massenkonzentration der alkalischen Knochenphosphatase wurde mit einem immunradiometrischen Verfahren bestimmt, diejenige von Osteocalcin mit einer immunenzymometrischen Methode, die sowohl das ,intakte“ Molekül als auch das Fragment (143) erfaßt. Die Patienten wurden mittels ${ }^{99 \mathrm{~m} T c-M e-}$ thylenbisphosphonat-Knochen-Szintigraphie charakterisiert. Gemäß der Z-score-Analyse ist die „diskriminatorische" Leistungsfähigkeit der alkalischen Knochenphosphatase derjenigen von Osteocalcin überlegen, wenn man diejenigen Tumorpatienten mit nach szintigraphischen Kriterien möglicher bzw. wahrscheinlicher ossärer Metastasierung mit solchen ohne pathologischen Befund im Szintigramm vergleicht ( $p$ $<0,05$ ). Zwischen alkalischer Knochenphosphatase und Osteocalcin bestand eine statistisch signifikante Korrelation ( $p<0,05 ; r=+0,626$ ). Die Receiver-operating-characteristic-Analyse zur Diskriminierung von Tumorpatienten mit und ohne Knochenmetastasen ergab einen ",accuracy"-Wert (= Fläche unter der ROC-Kurve) von 0,912 $\pm 0,074$ (Mittelwert \pm SE) für die alkalische Knochenphosphatase sowie von 0,687 \pm 0,086 für Osteocalcin $(p<0,05)$. Zusammenfassend läßt sich sagen, daß die Bestimmung der alkalischen Knochenphosphatase derjenigen von Osteocalcin bei der Diagnose von Knochenmetastasen vorgezogen werden sollte. .

Schlüsselwörter: Alkalische Phosphatase/Serum; Isoenzyme/Serum; Knochenmetastasen; Sensitivität und Spezifität; Osteocalcin/Serum; Szintigraphie.

Summary: In 73 tumour patients we compared the diagnostic validity of two parameters of bone formati-

\footnotetext{
'Institut für Klinische Chemie und Laboratoriumsdiagnostik, Heinrich-Heine-Universität Düsseldorf

2Korrespondenz-Addresse: Dr. med. Wolfgang Withold, Institut für Klinische Chemie und Laboratoriumsdiagnostik, Heinrich-HeineUniversität Düsseldorf, Moorenstraße 5, D-40225 Düsseldorf. Fax: $+49-211-81-18013$

Eingegangen: 15. Januar 1997 / Angenommen: 25. Februar1997
}

on (i.e. osteocalcin and bone alkaline phosphatase). Mass concentrations of bone alkaline phosphatase were determined with an immunoradiometric assay, whereas osteocalcin was measured employing an immunoenzymometric method. The latter assay measures both the 'intact' molecule and osteocalcin fragment (143). Patients were characterized by ${ }^{99 \mathrm{~m}} \mathrm{Tc}$-methylene bisphosphonate bone scintigraphy. According to $\mathrm{Z}$ score analysis bone alkaline phosphatase shows a higher discriminative power than osteocalcin when tumour patients with and without scintigraphic evidence of bone metastases were compared $(p<0.05)$. There was a statistically significant correlation between bone alkaline phosphatase values and serum osteocalcin concentrations ( $p<0.05 ; r=+0.626$ ). Receiver-operating characteristic analysis (discriminating tumour patients with and without bone metastases) yielded accuracy values of $0.912 \pm 0.074$ (mean \pm SE) (bone alkaline phosphatase) and $0.687 \pm 0.086$ (osteocalcin) ( $p$ $<0.05$ ). In conclusion, serum bone alkaline phosphatase should be preferred to osteocalcin determinations in the diagnostics of metastatic spread into bone.

Keywords: Alkaline Phosphatase/serum; Isoenzymes/ serum; Bone Neoplasms/secondary; Sensitivity and Specificity; Osteocalcin/serum; Radionuclide Imaging.

D ie alkalische Knochenphosphatase ist ein Ektoenzym, das in der Plasmamembran osteoblastärer Zellen lokalisiert ist. Methoden zur spezifischen Bestimmung der alkalischen Knochenphosphatase umfassen (a) die elektrophoretische Trennung, (b) die Präzipitation der alkalischen Knochenphosphatase mittels eines Lektins aus Triticum vulgare sowie (c) verschiedene immunologische Verfahren, unter denen gegenwärtig ein immunradiometrisches Verfahren sowie ein enzymatisches Verfahren nach Immunadsorption der alkalischen Knochenphosphatase in Mikrotiterplatten für Routine-Zwecke geeignet ist. Osteocalcin ist ein Polypeptid aus 49 Aminosäuren $\left(\dot{M}_{r}=6559\right)$, das von Osteoblasten in Abhängigkeit von Vitamin $\mathrm{K}$ und $\mathrm{Cal}-$ citriol synthetisiert wird. Nach der Sekretion wird Osteocalcin entweder in die extrazelluläre Matrix des Knochens inkorporiert oder in die Zirkulation abgege- 
ben. Osteocalcin unterlicgt intra- und extranssär ciner proteolytischen Degradation. Die hicrbei entstehenden Fragmente umfassen die Aminosäuren-Sequenzen 119. 20-43, 44-49, 20-49 und 1-43, wobei letzteres Fragment in der Regel quantitativ überwiegt (Übersicht bei l.c. $(1)$.

Beide klinisch-chemische Melggrößen gelten als Parameler des Knochenambaus bzw. -umsaltzes. In der vorliegenden Arbeit untersuchten wir vergleichend die diagnostische Validität cines immunradiometrischen Verfahrens zur Bestimmung der Massenkonzentration der alkalischen Knochenphosphatase sowie eines immunenzymoinetrischen Verfahrens zur Bestimmung von Osteocalcin bei der Diagnose von Knochenmetastasen.

\section{Materialien und Methoden}

\section{Patienten}

IVir untersuchten sowohl venöse Blutproben als auch den zweiten morgendlichen Spontanurin von 73 Patienten (29 Männer und 44 Frauen; Bereich des Lebensalters: 27 - 78 Jahre) mit malignen Tumoren. Die Probenahme erfolgte zwischen 8.00 und 10.00 Uhr vormittags. Die Proben wurden innerhalb von $4 \mathrm{~h}$ zentrifugiert und bis zur Analyse bei $-20^{\circ} \mathrm{C}$ eingefroren.

Folgende Primärtumor-Lokalisationen lagen vor: Mamma $(n=23)$, Lunge $(n=13)$, Prostata $(n=3)$, Niere $(n=2)$, Schilddrüse $(n=2)$, Ösophagus $(n=3)$, Uterus ( $(n=2)$, Kolon $(n=4)$, Pharynx $(n=1)$, Zunge $(n=1)$, Magen $(n=1)$, Tonsillen $(n=1)$, malignes Melanom $(\mathrm{n}=11)$, Hodgkins Lymphom $(\mathrm{n}=2)$ und Non-Hodgkin-Lymphom $(n=4)$. Alle Patienten waren normokalzämisch.

Entsprechend den szintigraphischen Befunden (die zwei Ärzte für Nuklearmedizin ohne Kenntnis der korrespondierenden biochemischen Befunde unabhängig voneinander erhoben) wurden die Patienten folgendermaßen eingeteilt: (a) Es liegen keine pathologischen szintigraphischen Befunde vor (Gruppe $0 ; n=23$ ). (b) Gemäß den erhobenen szintigraphischen Befunden erscheint das Vorliegen einer ossären Metastasierung unwahrscheinlich (Gruppe 1; $\mathrm{n}=29$ ), möglich (Gruppe 2; $n=9$ ) bzw. wahrscheinlich (Gruppe 3; $n=12$ ).

Bestimmung der Massenkonzentration der alkalischen Knochenphosphatase im Serum

Die Massenkonzentration der alkalischen Knochenphosphatase wurde mit einem immunradiometrischen Verfahren bestimmt (Tandem ${ }^{\circledR}-\mathrm{R}$ Ostase ${ }^{\mathrm{TM}}$; Hybritech Inc., San Diego, CA [U.S.A.]; Nr. 3040 BE). Die Methode bedient sich zweier Antikörper gegen verschiedene Epitope der alkalischen Knochenphosphatase. Die Kalibrationskurve wurde durch Punkt-zu-PunktInterpolation erstellt. Bei offenbar gesunden prämenopausalen Frauen $(n=40$; Bereich des Lebensalters:

Nicht standardisierte Abkürzungen: ELISA, enzyme-linked immunosorbent assay; ROC, receiver operator characteristic(s).
20 - 49 Jahre) fand sich folgendes Refcrenzintervall (2.5. - 97.5. Perzentile): 2.7 - $15.6 \mu \mathrm{g} / \mathrm{l}$.

\section{Bestimmung der Konzentration von Osteocalcin im Serum}

Die Konzentration von Osteocalcin wurde mit einem immunenzymometrischen Verfahren bestimmt (NMID hOstcocalcin ELISA; Osteometer BioTech A/S, Herlev, Dänemark; Version 3.0). Dic Methode bedient sich zweicr Antikörper gegen dic Aminosäuren-Sequenzen (7-19) und (20-43) von humanem Osteocalcin, so daß sowohl „intaktes" Osteocalcin (1-49) als auch das $\mathrm{N}$-terminal-midregionale Fragment (1-43) erfaßt wird. Die Kalibrationskurve wurde durch Punktzu-Punkt-Interpolation erstellt. Bei offenbar gesunden prämenopausalen Frauen (s.o.) fand sich folgendes Referenzintervall $(2,5$. - 97,5. Perzentile): $2-31 \mu \mathrm{g} / 1$.

\section{Bestimmung der Urin-Ausscheidung von immunreak- tivem Pyridinolin}

Die Bestimmung der Urin-Ausscheidung von immunreaktivem Pyridinolin erfolgte mit dem Collagen ${ }^{\mathrm{TM}}$ Crosslinks Kit (Metra Biosystems, Mountain View, CA [U.S.A.]; Nr. 8001). Dieser-kompetitive Immunoassay bedient sich eines polyklonalen Antikörpers gegen Pyridinolin (mit 100\%iger Kreuzreaktivität zu Desoxypyridinolin) und erfaßt neben der "freien" Fraktion der „cross-links“ die Peptid-gebundene (bis zu einer relativen Molekülmasse von 10 000). Die Kalibrationskurve wurde mit der 4-Parameter-Gleichung erstellt. Die Angabe der Werte erfolgt in $\mu$ mol Pyridinolin-Äquivalente/mol Kreatinin. Bei offenbar gesunden prämenopausalen Frauen (s.o.) fand sich folgendes Referenzintervall (2,5. - 97,5. Perzentile): 9 - 94 $\mu \mathrm{mol} / \mathrm{mol}$.

\section{Statistische Analyse}

Die eingesetzten statistischen Methoden umfassen den U-Test nach Wilcoxon, Mann \& Whitney (zweiseitig) für ungepaarte Variablen und lineare Regressions-Koeffizienten. Die „diskriminatorische“ Leistungsfähigkeit wurde durch Berechnung der „Z-Score-Werte“ (siehe Resultate) bestimmt. Die Bestimmung des ,accuracy"-Wertes (Mittelwert \pm Standardfehler) mittels ROC-Analyse erfolgte nach einem bereits früher von uns beschriebenen Verfahren [2].

\section{Ergebnisse}

Abbildung 1 zeigt die Verteilung der Werte der untersuchten biochemischen Marker des Knochenstoffwechsels bei den verschiedenen Patientengruppen. Gemäß der Z-score-Analyse ist die ,diskriminatorische" Leistungsfähigkeit der alkalischen Knochenphosphatase derjenigen von Osteocalcin überlegen, wenn man die Gruppen 2 und 3 mit Gruppe 0 vergleicht $(p<0,05)$ (Tab. 1).

Zwischen den untersuchten KnochenstoffwechselParametern (alkalische Knochenphosphatase, Osteo- 
Tabelle 1 Z-score-Analyse der untersuchten Knochenstoffwechsel-Marker bei Tumorpatienten mit und ohne ossäre Metastasierung.

\begin{tabular}{|c|c|c|c|}
\hline & Z-Score ${ }^{a}$ & & \\
\hline .. & $\begin{array}{l}\text { Alkalische Knochen- } \\
\text { phosphatase }[\mu \mathrm{g} / \mathrm{L}]\end{array}$ & Osteocalcin $[\mu g / L]$ & $\begin{array}{l}\text { Immunreaktives } \\
\text { Pyridinolin }[\mu \mathrm{mol} / \mathrm{mol}]\end{array}$ \\
\hline Gruppe ' 1 ' vs. Gruppe '0' & $+0,23$ & $+0,15$ & $+0,22$ \\
\hline Gruppe '2' vs. Gruppe '0' & $+2,64^{\star \star}$ & $+0,49$ & $+1,15^{\star *}$ \\
\hline Gruppe '3' vs. Gruppe '0' & $+7,53^{\star \star \star}$ & $+1,81^{* *}$ & $+1,95^{\star \star *}$ \\
\hline
\end{tabular}

a $\quad$ Z-score $=\left(\right.$ Mittelwert $_{\text {Gruppe } X}-$ Mittelwert $\left._{\text {Gruppe 0 }}\right) / S_{\text {SD }}$ Gppe o $(X=1,2,3)$

* $p<0,05$

$\star * * \quad p<0,01$

*** $p<0,001$ (jeweils im Vergleich zu Gruppe 0)

Tabelle 2 Korrelations-Koeffizienten für die Beziehung zwischen den untersuchten Knochenstoffwechsel-Markern bei Patienten mit unterschiedlicher Wahrscheinlichkeit einer ossären Metastasierung (nach szintigraphischen Kriterien).

\begin{tabular}{|llll} 
Patienten-Gruppe & A vs. B & A vs. C & B vs. C \\
Alle.Patienten & $+0,626^{*}$ & $+0,563^{\star}$ & $+0,454^{\star}$ \\
\hline${ }^{\prime}$ & $+0,052$ & $+0,039$ & $+0,350$ \\
$' 1^{\prime}$ & $+0,491^{\star}$ & $+0,058$ & $+0,133$ \\
'2' & $+0,414$ & $+0,164$ & $-0,119$ \\
'3' & $+0,603^{\star}$ & $+0,622^{*}$ & $+0,478$ \\
\hline
\end{tabular}

$A$, alkalische Knochenphosphatase

$B$, Osteocalcin

C, immunreaktives Pyridinolin

$\approx p<0,05$

calcin und immunreaktives Pyridinolin) bestanden statistisch signifikante Korrelationen ( $p<0,05 ; 0,45 \leq r$ $\leq 0,63$ ), wenn alle untersuchten 73 Patienten in die Analyse eingingen (Tab. 2,.Abb. 2 a,b).

Für die Receiver-operating-characteristic-Analyse (,ROC") wurde zwischen den Gruppen 2 und 3 einerseits und den Gruppen- 0 und 1 andererseits unterschieden. Die Entscheidungsschwellén entsprachen der 10., 20., ..., 90. Perzentile der jeweiligen Werte in der Gesamtheit aller 73 untersuchten Patienten. Der „accuracy"-Wert (= Fläche unter der ROC-Kurve) betrug $0,912 \pm 0,074$ für die alkalische Knochenphosphatase und $0,687 \pm 0,086$ für Osteocalcin $(p<0,05)$ (Abb. 3).

\section{Diskussion}

In einer Studie von Berruti et al. [3] ist zwar bei Patientinnen mit Mammakarzinom und Knochenmetastasen sowohl die alkalische Knochenphosphatase als auch Osteocalcin gegenüber solchen Patientinnen mit ,fortgeschrittener" Erkrankung (ohne Knochenmeta- stasen) bzw. Metastasen in andere Organe erhöht. doch erreicht die Differenz nur für die alkalische Knochenphosphatase das Signifikanz-Niveau. Bei osteoblastischen Metastasen weist Osteocalcin einen prozentualen Anstieg von lediglich $76 \%$ gegenüber Tumorpatienten ohne Knochenmetastasen auf, alkalische Knochenphosphatase jedoch von $1200 \%$ (!) [4]. Kamby et al. [5] berichten, daß die diagnostische Effizienz der Gesamt-Aktivität der alkalischen Phosphatasen derjenigen von Osteocalcin überlegen ist, wobei weder das Ausmaß noch die Zahl der ossären Metastasen eine Beziehung zu den Osteocalcin-Konzentrationen aufweist. Shih et al. [6] führten bei 9 Patienten mit nicht-erhöhten Osteocalcin-Werten den szintigraphischen Nachweis von Knochenmetastasen. Möglicherweise ist die unterlegene diagnostische Validität (im Vergleich zur alkalischen Knochenphosphatase) bezüglich des Nachweises einer ossären Metastasierung ein allgemeines Merkmal von Knochenmatrix-Proteinen. Wir beobachteten eine ähnliche Unterlegenheit gegenüber alkalischer Knochenphosphatase für ein phosphoryliertes Glykoprotein der Knochenmatrix, das „,bone sialoprotein" [7].

Darüber hinaus weist die Bestimmung von Osteocalcin - im Vergleich zur alkalischen Knochenphosphatase - eine Reihe von Nachteilen auf (1): (a) Osteocalcin zeichnet sich durch einen ausgeprägten zirkadianen Rhythmus mit morgendlichen Maxima aus. (b) Bereits geringfügige Einschränkungen der glomerulären Filtrationsrate führen - Retentions-bedingt - zu erhöhten Werten. (c) Die mit verschiedenen Verfahren bestimmten Werte weichen voneinander deutlich ab; dies ist nicht nur auf eine unterschiedliche FragmentSpezifität der zur Verfügung stehenden Assays zurückzuführen, sondern auch auf Unterschiede in der Kalibration der Verfahren, da ein verbindliches Referenzmaterial zum gegenwärtigen Zeitpunkt (noch) nicht zur Verfügung steht. (d) Osteocalcin weist vielfach aufgrund einer auch in vitro stattfindenden Proteolyse - eine ausgeprägte präanalytische Instabilität auf.

Das von uns eingesetzte Verfahren zur Bestimmung von Osteocalcin zeichnet sich zwar durch befriedigende präanalytische Stabilität aus [8], da das quantitativ 

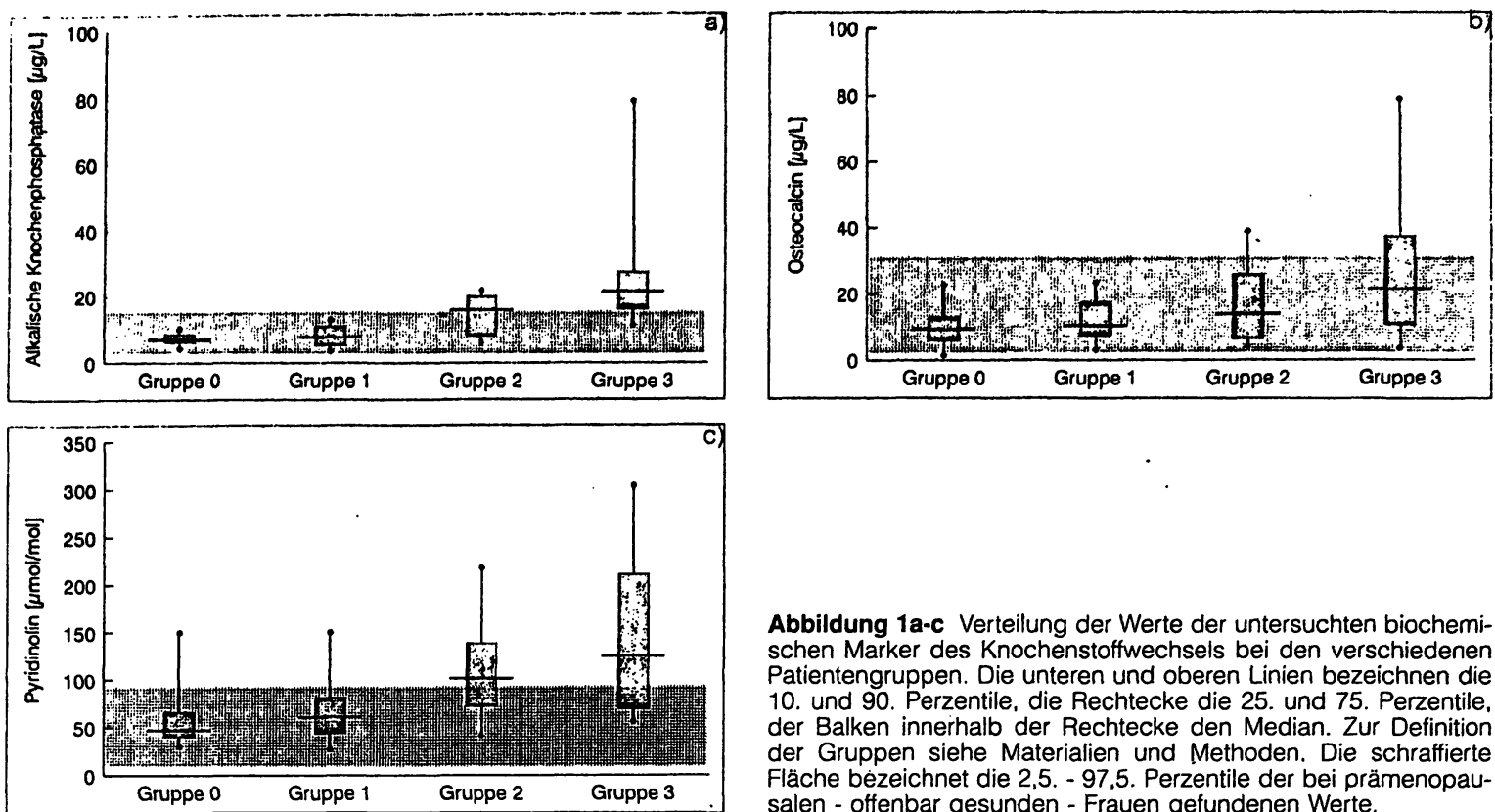

Abbildung 1a-c Verteilung der Werte der untersuchten biochemischen Marker des Knochenstoffwechsels bei den verschiedenen Patientengruppen. Die unteren und oberen Linien bezeichnen die 10. und 90. Perzentile, die Rechtecke die 25. und 75. Perzentile, der Balken innerhalb der Rechtecke den Median. Zur Definition der Gruppen siehe Materialien und Methoden. Die schraffierte Fläche bezeichnet die 2,5. - 97,5. Perzentile der bei prämenopausalen - offenbar gesunden - Frauen gefundenen Werte.
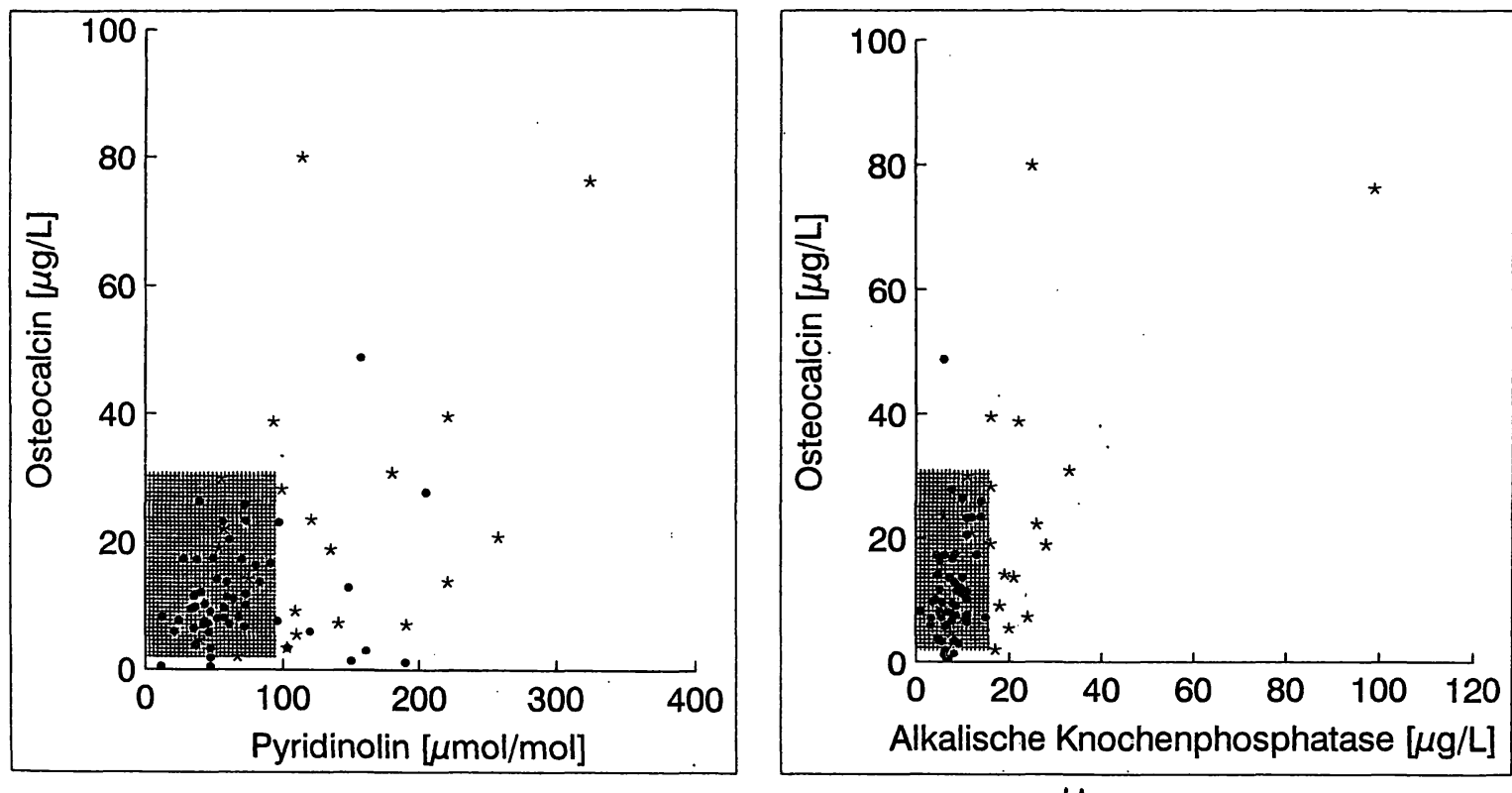

Abbildung 2a,b Beziehung zwischen den Knochenstoffwechsel-Parametern bei 73 Tumorpatienten. Die den Gruppen 2 und 3 angehörenden Patienten sind durch Sterne symbolisiert, die übrigen Patienten durch Punkte. Die schraftierte Fläche umfaßt das bei prämenopausalen Frauen gefundene Referenzintervall.

wichtigste Osteocalcin-Fragment (1-43) bei dieser Methode miterfaßt wird. Dennoch ist nach unseren Untersuchungen diese Meßgröße der alkalischen Knochenphosphatase hinsichtlich der diagnostischen Validität unterlegen, so daß letzterer Parameter bei der Diagnose von Knochenmetastasen bevorzugt werden sollte. Hierbei ist die spezifische Bestimmung der alkalischen
Knochenphosphatase der Gesamt-Aktivität der alkalischen Phosphatasen hinsichtlich der mittels Z-scoreAnalyse ermittelten "diskriminatorischen" Leistungsfähigkeit überlegen [9]. Bei Patienten mit metastasierendem Prostatakarzinom zeigt die alkalische Knochenphosphatase bei gleicher diagnostischer Empfindlichkeit eine höhere diagnostische Spezifität als das 


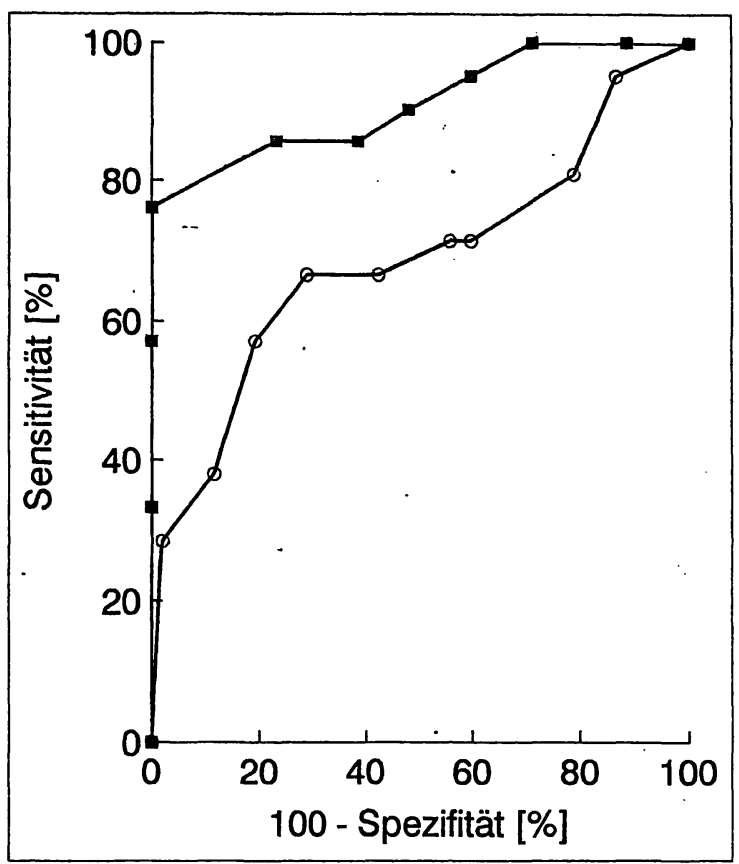

Abbildung 3 ROC-Analyse von alkalischer Knochenphosphatase und Osteocalcin zur Diskriminierung zwischen Tumorpatienten mit (= Gruppen 2 und $3 ; n=21$ ) und ohne Knochenmetastasen (= Gruppen 0 und $1 ; n=52$ ). Die alkalische Knochenphosphatase wird durch Quadrate, Osteocalcin durch Kreise symbolisiert. Die Flächen unter den Kurven betragen 0,912 $\pm 0,074$ (alkalische Knochenphosphatase) und $0,687 \pm 0,086$ (Osteocalcin) $(p<0,05)$.

Prostata-spezifische Antigen [10]. Auch bei der Verlaufskontrolle dieser Patientengruppe erweist sich die Bestimmung der alkalische Knochenphosphatase als nützlich [11]. Demgegenüber zeichnet sich das carboxyl-terminale Propeptid von Typ-I-Prokollagen, ein weiterer Parameter des Knochenanbaus, i.d.R. durch eine niedrige diagnostische:Sensitivität aus. Dies triftt auch auf Patienten mit Knochenmetastasen zu [12].

Im Gesamt-Kollektiv der von uns untersuchten $\mathrm{Pa}$ tienten fand sich eine-signifikante Korrelation zwischen Osteocalcin und alkalischer Knochenphosphatase - im Gegensatz zu dem von Burlina et al. [13] berichteten Fehlen einer solchen Korrelation. Hierbei ist der Charakter der Knochenmetastasen (osteoplastisch vs. -lytisch) zu berücksichtigen. Berruti et al. [4] fanden nur bei osteoplastischen Metastasen eine Korrelation zwischen alkalischer Knochenphosphatase und Osteocalcin. Auch bei Patientinnen mit postmenopausaler Osteoporose findet sich mitunter keine signifikante Korrelation zwischen den beiden Knochenanbau-Parametern [14].

Als klinisch-chemische Meßgröße der Wahl zur Erfassung der Knochenresorption gilt derzeit die UrinAusscheidung der „pyridinium cross-links“ (Pyridinolin, Deoxypyridinolin) - Quervernetzungs-Produkte des Knochenkollagens. Mammakarzinom-Patientinnen mit Knochenmetastạsen weisen signifikant höhere Urin-Ausscheidungs-Werte auf als solche ohne Nachweis von Knochenmetastasen [15]. Bei Patienten mit Prostatakarzinom korreliert die Urin-Ausscheidung der "cross-links" mit der Tumormasse [16], die klinische Progression kann mit Hilfe eines Anstiegs der ,crosslinks"-Werte eher vorausgesagt werden als mittels Knochenszintigraphie [17]. Es muß jedoch betont werden, daß eine erhöhte Ausscheidung von ,pyridinium cross-links" vielfach auch bei Patienten ohne szintigraphischen Nachweis von Knochenmetastasen gefunden werden kann. Dies läßt sich u.a. auf folgende Ursachen zurückführen: (a) eine Immobilisierung der $\mathrm{Pa}$ tienten, (b) humorale Faktoren, von denen bekannt ist, $\mathrm{da}$ ß sie die Knochenresorption steigern können (z.B. Parathyroid hormone-related protein, Interleukin-1. Tumor-Nekrose-Faktoren), (c) eine unzureichende diagnostische Sensitivität des Knochenszintigramms (insbesondere für osteolytische Metastasen), (d) eine Freisetzung von ,pyridinium cross-links“ aus extraossärem - vom Tumor infiltrierten - Gewebe [18]. Die von uns gefundene Korrelation zwischen Pyridinolin und den untersuchten Knochenanbau-Parametern ist als Hinweis auf ein erhaltenes "coupling" zwischen An- und Abbau zu interpretieren [19].

\section{Literatur}

1. Withold W. Monitoring of bone turnover: biological, preanalytical and technical criteria in the assessment of biochemical markers [review]. Eur J Clin Chem Clin Biochein 1996; 34:785-99.

2. Withold W, Schulte U, Reinauer H. Method for determination of bone alkaline phosphatase activity: analytical performance and clinical usefulness in patients with metabolic and malignant bone diseases. Clin Chem 1996; 42:210-7.

3. Berruti A, Torta M, Piovesan A, Raucci CA, Orlandi F, Panero A. Dogliotti L, Angeli A. Biochemical picture of bone metabolism in breast cancer patients with bone metastases. Anticancer Res $1995 ; 15: 2871-5$.

4. Berruti A, Piovesan A. Torta M, Raucci CA. Gorzegno G, Paccotti P, Dogliotti L, Angeli A. Biochemical evaluation of bone turnover in cancer patients with bone metastases. Relationship with radiograph appearances and disease extension. Br J Cancer 1996; 73:1581-7.

5. Kamby C, Egsmose C, Soletormos G, Dombernowsky P. The diagnostic and prognostic value of serum bone Gla protein (osteocalcin) in patients with recurrent breast cancer. Scand J Clin Lab Invest 1993: 53:439-46.

6. Shih WJ, Collins J, Wierzbinski B, Magoun S, Ryo UY. ${ }^{99 \mathrm{~m}} \mathrm{Tc}$ HMDP bone scintigraphy findings in association with concurrent serum osteocalcin measurements. Radiat Med 1991; 9:162-6.

7. Withold W, Armbruster FP, Karmatschek M. Reinauer H. Bone sialoprotein in serum of patients with malignant bone diseases. Clin Chem 1997; 43:85-91.

8. Rosenquist C, Qvist P. Bjarnason N. Christiansen C. Measurement of a more stable region of osteocalcin in serum by ELISA with two monoclonal antibodies. Clin Chem 1995; 41:1439-45.

9. Withold W. Georgescu G, Khakzad H, Vosberg H, Mueller-Gaertner HW, Reinauer H. Efficacy of simultaneous determination of bone alkaline phosphatase mass concentration in serum and pyridinium cross-links for detection of bone metastases. Clin Biochem 1995; 28:511-8.

10. Wolff JM, Jttel T, Boeckmann W, Reinike T, Habib FK, Jakse G. Skeletal alkaline phosphatase in the metastatic workup of patients with prostate cancer. Eur Urol 1996; 30:302-6.

11. Lorente JA, Morote J, Raventos C. Encabo G. Valenzuela H. Clinical efficacy of bone alkaline phosphatase and prostate specific antigen in the diagnosis of bone metastasis in prostate cancer. J Urol 1996; 155:1348-51. 
12. Withold W, Georgescu G, Khakzad H, Vosberg H, Mueller-Gacrtner HW, Reinaucr H. Simultancous assessment of bone collagen synthesis and degradation in patients with different malignant tumours: comparison with the results of $99 \mathrm{~m} \mathrm{Tc}$-methylene bisphosphonate bone scintigraphy. Eur J Clin Chem Clin Biochem 1995; 33:479-85.

13. Burlina A, Rubin D, Secchiero S, Sciacovclli L, Zaninotto M, Plebani M. Monitoring skeletal cancer metastases with the bone isocnzyme of tissue unspecific alkaline phosphatase. Clin Chim Acta 1994; 226:151-8.

14. Diego EMD, Martin MAD, Delapiedra C, Rapado A. Lack of correlation between levels of osteocalcin and bone alkaline phosphatase in healthy control and postmenopausal osteoporotic women. Horm Metab Res 1995; 27:151-4.

15. Massidda B, Ionta MT, Foddi MR, Mascia L, Bruder F, Aloi MB, Meleddu C, Giannoni MN. Usefulness of pyridinium crosslinks and CA 15-3 as markers in metastatic bone breast carcinoma. Anticaner Res 1996; 16:2221-4.
16. Ikeda I, Miura T, Kondo I. Pyridinium cross-links as urinary markers of bone metastases in patients with prostate cancer. $\mathrm{Br} \mathrm{J}$ Urology 1996; 77:102-6.

17. Takeuchi S-I, Kyouko A, Saitoh H, Yoshida K-I, Miura M. Urinary pyridinoline and deoxypyridinoline as potential markers of bone metastasis in patients with prostate cancer. J. Urology 1996; 156:1691-5.

18. Pecherstorfer M, Zimmer-Roth I, Schilling T, Woitge HW, Schmidt H, Baumgartner G, Thiebaud D. Ludwig H, Seibel M. The diagnostic value of urinary pyridinium cross-links of collagen, serum total alkaline phosphatase, and urinary calcium excretion in neoplastic bone disease. J Clin Endocrinol Metab 1995; 80:97-103. 19. Paterson CR, Robins SP, Horobin JM, Preece PE, Cuschieri A. Pyridinium crosslinks as markers of bone resorption in patients with breast cancer. Br J Cancer 1991; 64:884-6. 